\title{
Basal mTORC2 activity and expression of its components display diurnal variation in mouse perivascular adipose tissue
}

Katja Drägert ${ }^{1,2}$, Indranil Bhattacharya ${ }^{1,2}$, Michael N. Hall ${ }^{3}$, Rok Humar ${ }^{1,2}$, Edouard Battegay $^{1,2,4}$, Elvira Haas ${ }^{1,2}$

${ }^{1}$ Research Unit, Department of Internal Medicine, University Hospital Zurich

${ }^{2}$ Center of Competence Multimorbidity and University Research Priority Program "Dynamics of Healthy Aging", University of Zurich

${ }^{3}$ Biozentrum, University of Basel

${ }^{4}$ Zurich Center for Integrative Human Physiology, University of Zurich

All Switzerland

Correspondence to: Elvira Haas, Research Unit, Department of Internal Medicine University Hospital Zurich, Wagistrasse 12, 8952 Schlieren, Switzerland

Email: Elvira.haas@usz.ch,Phone: +41 44 5563230, Fax:+41 445563232 


\section{Abstract}

In adipose tissue mTOR complex 2 (mTORC2) contributes to the regulation of glucose/lipid metabolism and inflammatory molecule expression. Both processes display diurnal variations during the course of the day. RICTOR and mSIN1 are unique and essential components of mTORC2, which is activated by growth factors including insulin.

To assess whether mTORC2 components display diurnal variations, we analyzed steady state mRNA expression levels of Rictor, $m \operatorname{Sin} 1$, and $m$ Tor in various adipose tissues during a 24 hours period. Diurnally regulated expression of Rictor was detected in brown adipose tissues displaying highest mRNA expression levels at the beginning of the 12 hour light period (zeitgeber time 2, ZT2). Gene expression patterns of $m \operatorname{Sin} 1$ and $m$ Tor displayed a similar diurnal regulation as Rictor in PVAT while smaller changes were detected for these genes in aorta during the course of the day. Basal mTORC2 activity was measured by phosphorylation of protein kinase C (PKC) a at serine 657 was higher at ZT14 as compared with ZT2 in PVAT. In line, gene expression of inflammatory molecules nitric oxide synthase 2 and tumor necrosis factor $\alpha$ was lower at ZT 14 compared to ZT2

Our findings provide evidence for a diurnal regulation of expression of mTORC2 components and activity. Hence, mTORC2 is possibly an integral part of diurnally regulated signaling pathways in PVAT and possibly in other adipose tissues. 
Key words: mTORC2, Rictor, diurnal, mSIN1, PKCalpha

Abbreviations: mTOR, mammalian target of rapamycin; mTORC2, mTOR complex 2; ZT, zeitgeber time; PKC, protein kinase C; TNFa, tumor necrosis factor $\alpha$; NOS2, inducible nitric oxide synthase 2, PVAT, perivascular adipose tissue; BAT, brown adipose tissue; EFAT, epididymal fat. 


\section{Introduction}

The circadian system, an endogenous time-keeping mechanism, facilitates behavioral and physiologic adaptation to daily environmental cycles to maintain energy balance, thus ensuring survival [1]. Clock genes encode the circadian clock which is hierarchically organized: the light responsive master clock is located in the suprachiasmatic nucleus whereas peripheral clocks are present in nearly all cells of the body. While the master clock entrains and synchronizes the peripheral clocks with the day night cycle, peripheral clocks are additionally entrained by feeding and behavioral signals to regulate tissue specific programs in an anticipatory manner [2, 3].

Misalignment of the circadian system with the diurnal variation of resting/activity and feeding/fasting cycles results in metabolic dysfunction and increased morbidity [3]. Adipose tissue is a metabolically highly active organ and is actively involved in the integration of fasting/feeding periods into the whole organism [4]. Adipocyte-specific deficiency of mTORC2 by ablation of Rictor results in higher lean mass due to increased levels of insulin and insulin-like growth factor, enhanced glucose metabolism and insulin resistance in mice $[5,6]$. Using this mouse model we have recently shown that clock gene expression in perivascular adipose tissue (PVAT) is altered in comparison with tissue derived from control mice [7]. Deletion of Rictor in adipocytes had no effect on aortic tissue consisting mainly of smooth muscle and endothelial cells. These alterations in gene expression were associated with changes in diurnal blood pressure regulation.

Mammalian target of rapamycin (mTOR) is a ubiquitously expressed serine/ threonine kinase which is present in two multi-protein complexes: mTOR complex 1 (mTORC1) and complex 2 (mTORC2) [8, 9]. mTORC2 contains RICTOR and mSIN1 as unique components. The presence of RICTOR [10], the rapamycin-insensitive 
companion of mTOR, and mSIN1 [11] are sine qua non for mTORC2 to propagate phosphoinositid-3-kinase elicited signals originating from hormones and growth factors to regulate metabolic and other processes [12]. Here we hypothesized that molecules of the mTORC2 pathway in PVAT may be diurnally regulated to participate in anticipatory responses such as blood pressure regulation. 


\section{Materials and Methods}

\section{Mice and tissue collection}

In all experiments, male mice (18-23 weeks old) carrying a floxed Rictor gene were used as control mice (mice without the Cre transgene, Rictor ${ }^{f / f t}$, termed henceforth control). Adipocyte deletion of Rictor (Rictor ${ }^{2 P 2 K O}$ ) was accomplished by crossing Rictor $^{f / f t}$ mice with C57BL/6J mice expressing CRE recombinase under the control of the adipocyte protein 2 (aP2, also known as fatty acid binding protein 4) gene promoter (purchased from JAX Laboratories, Bar Harbor, Maine, USA).

Mice were kept in the institutional animal facilities (University of Zurich and University Hospital of Zurich, Switzerland) at $22{ }^{\circ} \mathrm{C}$ with a $12: 12$ hour light/dark cycle. Light onset was at 7 am [zeitgeber time (ZT) 0] and light offset was at 7 pm (ZT 12). Animals had access to standard chow (4.5\% calories from fat; Kliba Nafag, Kaiseraugust, Switzerland) and water ad libitum. All mice were genotyped for Rictor (standard PCR, forward primer 5'-3': TTATTAACTGTGTGTGGGTTG and reverse primer 5'-3' CGTCTTAGTGTTGCTGTCTAG) and Cre recombinase (quantitative RTPCR) using specific mouse primers listed in Table 1 and standard protocols (JAX Laboratories, Bar Harbor, Maine, USA). Before sacrifice, mice were weighed and anesthetized by intraperetoneal (i.p.) injection (xylazine: 20; ketamine: 100; and acepromazine: 3.0 ; in $\mathrm{mg} / \mathrm{kg}$ body weight) and exsanguinated via cardiac puncture. All mouse experiments described here were approved by the Kantonales Veterinaeramt of Zurich, Switzerland (License numbers ZH44/2011 and ZH184/2014). Therewith, the investigation conforms to the Guide for the Care and Use of Laboratory Animals published by the US National Institutes of Health (Edition, 2011). 


\section{RNA isolation, reverse transcription and quantitative real-time PCR}

The method was performed essentially as described earlier [7]: RNA from PVAT, EFAT and BAT was extracted using RNeasy ${ }^{\circledR}$ lipid tissue kit and from thoracic aortas using RNeasy ${ }^{\circledR}$ fibrous tissue kit, according to the protocol of the manufacturer (Qiagen, Hombrechtikon, Switzerland) respectively. DNA was removed using an oncolumn DNase digestion method (RNase-free DNase, Qiagen). Before reverse transcription, RNA quantity and quality was determined using Nanodrop 2000 Spectrophotometer and Agilent 2100 Bioanalyzer. Reverse transcription of $50 \mathrm{ng}$ RNA from PVAT, and thoracic aortas was performed using WT (Whole Transcript)Ovation ${ }^{\circledR}$ Pico RNA Amplification System (Nugen, Bemmel, Netherlands) and from EFAT and BAT using iScript ${ }^{\mathrm{TM}}$ Reverse Transcription Supermix according manufactures recommendations (BioRad, Hercules, CA, USA). For quantitative RT$\mathrm{PCR}$, final cDNA concentration was adjusted to $10 \mathrm{ng}$ in a $15 \mu \mathrm{l}$ reaction volume. Each reaction was performed in duplicates using Bio-Rad CFX96 Real-Time System and $\mathrm{iQ}^{\mathrm{TM}} \mathrm{SYBR}^{\circledR}$ Green Supermix (BioRad, Hercules, CA, USA) and specific mouse primers. Gene expression in PVAT, thoracic aortas, EFAT and BAT was normalized to the reference gene acidic ribosomal phosphoprotein (Arbp), using the comparative $C(T)$ method [13]. If not otherwise noted, mouse primer pairs for selected genes were designed using Primer Blast (NCBI, USA) [14] and are listed in Table 1.

\section{SDS-PAGE and Western blot analysis}

Tissues were homogenized in tissue lysis buffer using Tissue Lyser (Qiagen, Hombrechtikon, Switzerland): 6 glass beads $(3 \mathrm{~mm})$ were used per $2 \mathrm{ml}$ safe-lock reaction. Tissue lysis buffer constituted of $500 \mathrm{mM}$ Tris ( $\mathrm{pH}$ 7.5), 2 mM EDTA, 2 mM EGTA, $1 \%$ Triton X, $10 \%$ Glycerol, proteinase/ phosphatase inhibitor (Roche, Switzerland). For further processing, tissue extracts were passed 4 times through a 
20-G needle, centrifuged ( $10 \mathrm{~min}, 10000 \times \mathrm{g}, 4^{\circ} \mathrm{C}$ ) and the supernatant transferred to a new $1.5 \mathrm{ml}$ reaction tube. Protein concentration was determined using Quick Start ${ }^{\mathrm{TM}}$ Bradford Protein Assay according manufactures recommendations and extracts were diluted in sample buffer (4\% SDS, $20 \%$ glycerol, $120 \mathrm{mM}$ Tris pH 6.8 and $5 \% \beta$-mercaptoethanol) to equal concentrations. Samples were heated $\left(95^{\circ} \mathrm{C}, 5\right.$ min). A reducing SDS-PAGE was loaded with $20 \mu \mathrm{g}$ total protein/well for subsequent Western blot analysis using a Criterion Precast Tris-HCl gel (Bio-Rad). Proteins were transferred to polyvinylidene difluoride membranes (Bio-Rad), which were blocked by incubating for $1 \mathrm{~h}$ at room temperature in $5 \%$ bovine serum albumin (BSA, Gibco Europe) in Tris-buffered saline with Tween-20 (TBS-T, $50 \mathrm{mM}$ Tris-Cl, pH 7.5. 150 $\mathrm{mM} \mathrm{NaCl}, 0.1 \%$ Tween-20 (Merck, Dietikon, Switzerland)) on an orbital shaker. Primary antibodies as listed in Table 2 were used for overnight incubation at $4^{\circ} \mathrm{C}$, diluted in TBS-T, supplemented with $5 \%$ BSA. Membranes were washed in TBS-T and appropriate HRP-conjugated secondary antibodies were incubated for 1 hour at room temperature. After 4 washing steps using TBS-T blots were developed with chemiluminescence Western blotting detection reagent (SuperSignal West Pico or Femto, Thermo Fisher) and analyzed using a Chemi Doc XRS system with Quantity One 1-D Analysis software, Version 4.5.0 (Bio-Rad).

\section{Statistical Analysis}

Comparisons between groups for other experiments were analyzed by two-tailed Student's t-test. Differences were considered statistically significant at values of $P<0.05$. Statistical analyses were performed using GraphPad Prism 5.04 program for Windows (GraphPad software, San Diego, CA, USA). 


\section{Results}

\section{Diurnal oscillations of Rictor gene expression in adipose tissue depots but not}

in aorta

To investigate whether Rictor gene expression is diurnally regulated in adipose tissue, we measured mRNA expression levels in various adipose tissue depots from control mice (Rictor ${ }^{f / f t}$ mice). Expression levels of Rictor in white adipose tissue depot derived from epididymal fat (EFAT) displayed no differences at the beginning of the light period (Zeitgeber time (ZT) 2, 9 am) compared to the beginning of the dark period (ZT 14, Fig. 1A). In brown adipose tissue derived from the interscapular region (BAT; Fig. 1B) and PVAT (Fig. 1C) mRNA expression levels of Rictor peaked at ZT2 (9 am) and then declined steadily with the progression of the day reaching lowest levels at ZT $18(1 \mathrm{am})$ and ZT 22 (5 am). Differences in Rictor expression during the 24 period amounted to 2.9 and 3.2-fold difference for BAT (Fig. 1B) and PVAT (Fig. 1C), respectively, while at ZT 2 compared to ZT 14 only in PVAT a significant difference was detected $(p<0.01)$. Aorta (Fig. 1D) displayed only very small variations in Rictor mRNA expression levels. As expected, in adipose Rictor knockout mice (Rictor ${ }^{\text {AP2KO }}$ mice) Rictor expression levels were low and remained nearly constant over time in all adipose tissue depots analyzed (Figure 1A-C).

\section{Diurnal variations of $m$ Tor and $m$ Sin1 gene expression levels in PVAT and aorta}

As we found the largest differences in expression levels in PVAT we focused on this adipose tissue depot and the aorta as reference. To examine whether the diurnal regulation of Rictor expression is unique within the mTORC2 signaling pathway we measured mRNA expression levels of $m \operatorname{Sin} 1$ (Fig 3A), another unique and essential 
component of mTORC2, and mTor (Fig. 2B) as the catalytic core of the two mTOR complexes. mRNA expression levels of mTor were similarly regulated in PVAT and aorta (Fig. 2A) with highest levels at ZT 6 (1 pm). We found a 3-fold overall variation of $m$ Tor expression during a 24 hour period (Fig. 2A). Interestingly, mSin 1 levels varied strongly in PVAT (5.7 fold) while in aorta expressional differences were minor (2.2 fold, respectively, Fig. 2B). Only in PVAT significant differences in expression levels of $m$ Tor $(\mathrm{p}=0.035)$ and $m \operatorname{Sin} 1(\mathrm{p}=0.022)$ were detected between the ZT 2 and 14.

\section{Diurnal regulation basal mTORC2 activation and inflammatory gene expression in PVAT is diurnally regulated}

Next, we examined whether gene expression variations of Rictor and $m \operatorname{Sin} 1$ translated in variations of protein expression. We determined protein expression levels of RICTOR, mSIN1 and mTORC2 downstream signaling proteins by examining phosphorylation status of AKT on serine 473 [15] and PKCa at serine 657 [16] at two different time points - ZT2 and ZT14 - to encompass the resting and active period of the mice, respectively. Protein expression of RICTOR $(p<0.05)$ and mSIN1 $(p<0.01)$ was higher at ZT14 as compared to ZT 2 (Fig. 3A). Accordingly, we detected stronger basal phosphorylation of PKCa at ZT14 compared with ZT2 $(p<0.01)$ indicating a higher basal activity of mTORC2 at this time point. Total PKCa protein expression levels were similar at ZT2 and ZT14. Basal serine 473-phosphorylation of AKT was not clearly distinguishable between the two time points analyzed as signal was very low.

PVAT contributes to the regulation of the vascular reactivity $[17,18]$ and we have shown previously that $\mathrm{mTORC} 2$ can regulate vascular contraction and dilation by affecting inflammatory molecule expression [19]. Thus, we speculated that 
inflammatory molecule expression is modulated in PVAT in a time-of-day-dependent matter. To test this, we measured gene expression of nitric oxide synthase 2 (Nos2) / inducible $N$ and tumor necrosis factor $\alpha(T n f \alpha)$ at ZT2 and 14. In PVAT, Nos2 and Tnfa gene expression levels were approximately $70 \%(p=0.068)$ and $90 \%(p<0.05)$ lower at ZT 14 compared to ZT2 suggesting a diurnal variation of inflammatory gene expression. 


\section{Discussion}

The present study strongly suggests that several time points during the 24 hour period need to be analyzed in complex animal models in order to display the regulatory circuits involving mTORC2 activity. We found that mTORC2 components and activity are diurnally regulated in PVAT. Diurnally regulated expression of Rictor was mainly detected in brown adipose tissues displaying highest mRNA expression levels at the beginning of the 12 hour light period (zeitgeber time 2, ZT2). Gene expression patterns of $m \operatorname{Sin} 1$ and $m$ Tor displayed a similar diurnal regulation as Rictor in PVAT. mSin1, another essential component besides Rictor of the mTORC2, is diurnally regulated in PVAT but not in aortic tissue at the gene expression level. These diurnal differences in expression are translated in higher basal activity of mTORC2 at ZT14 as shown by phosphorylation of PKCa at serine 657. Thus, the RICTOR- PKCa pathway may contribute to diurnal regulation of anticipatory physiologic processes such as inflammatory molecule expression in PVAT and possibly blood pressure regulation.

Anticipatory physiological regulation is an adaptive strategy that enables animals to respond faster to physiologic and metabolic challenges [20]. For example, diurnal oscillations in the secretion of many hormones (e.g. cortisol, leptin, ghrelin) allow an animal to be in the most appropriate physiological state at different time points of the day [20]. Blood pressure and vascular contractility - important parameters of vascular physiology - also follow circadian rhythms [21]. In addition, acute cardiovascular events such as heart attack and stroke occur most frequently in the morning [22] further strengthening the concept that diurnal regulation of the signaling pathways impacting vascular physiology. 
Interestingly, there is some evidence that components of the mTORC2 signaling pathway, phosphatidylinositol-3-kinase and its downstream kinase Akt are regulated by the circadian system in the vasculature [23]. In the present study, we found in PVAT that basal mTORC2 activity as displayed by PKCa phosphorylation was higher at ZT14 which corresponds to the beginning of active period of the mice compared to ZT2. What could be the physiological advantage of higher basal mTORC2 activity during the active period? The modulation of basal PKCa and possibly Akt phosphorylation by mTORC2 may contribute to fine tuning of the sensitivity to growth factors during the course of the day. Therewith, in periods of high metabolic activity such as at the beginning of the dark period, which corresponds to the beginning of the active period in mice, the mTORC2 signaling pathway may reduce inflammatory gene expression and, at the same time, enhance glucose metabolizing pathways. Indeed, glucose uptake is dependent on mTORC2 in brown fat cells [24] and a possible link between mTOR signaling, diurnal gene expression and metabolic regulation in adipose tissue has also been suggested [25]. Furthermore, tissue repair and immune responses are known to be fluctuating during the course of the day [26] which may be facilitated by diurnally regulated mTORC2 activity. Thus, the diurnal variation of basal mTORC2 activity might contribute to the regulation of glucose metabolism and inflammatory molecule expression in PVAT considered to consist mostly of brown adipocytes [27].

At the first glance, this is a contradictory result to the diurnal course of blood pressure: in mice blood pressure is low during the beginning of the resting period (ZT2 to ZT 6) and steadily rises to reach maximal values at ZT14 thereafter. A possible explanation is that locomotor activity, which accounts predominantly to the diurnal variations of blood pressure in mice [28], conceals the effects of shifting mTORC2 activity. We therefore speculate that during metabolic diseases such as 
obesity the diurnal variation of Rictor is dysregulated and perturbs vascular physiology. In line, we have shown that ablation of Rictor in adipose tissue and brain results in impaired PVAT function resembling a phenotype which is similar to metabolic disease and impaired diurnal blood pressure regulation possibly by affecting inflammatory molecule and natriuretic peptide expression $[7,19]$.

PKCa is involved in multiple cellular responses including cell survival, motility, and inflammation [29]. For example, in glioma and mammary epithelia cells mTORC2 signals via PKCa ensuring cell survival and motility [30, 31]. Similarly, overall cellular mTORC2 activity, affected by Rictor and $m \operatorname{Sin} 1$ expression, may impact PKCa pathway activation to contribute to the anticipatory regulation of blood pressure via regulation of inflammatory molecule or natriuretic peptide expression in PVAT. Yet to be defined mechanisms might also be involved.

Taken together, our findings suggest that mTORC2 may function as a diurnal rheostat in PVAT and possibly other adipose tissues by diurnal regulation of expression of mTORC2 components and activity. Better understanding of these regulatory circuits might refine strategies of patient treatment aiming at modulation of the mTORC2 pathway by optimal and efficient timing to alleviate metabolic and blood pressure disorders in patients.

\section{Acknowledgements}

The authors are grateful to all members of research unit, Internal Medicine, University Hospital Zurich for stimulating discussions and, in addition, to Ana Perez-Dominguez and Daniela Bukatz for their excellent technical assistance. 


\section{Sources of Funding}

This work was supported by funding from University of Zurich, University Hospital of Zurich, Hartmann Müller Stiftung, Forschungskredit of the University of Zurich (GrantNo. FK-13-026), the Swiss National Science Fundation, Stiftung Baugarten, and Jubiläumsstiftung der Schweizerischen Lebensversicherungs- und Rentenanstalt für Volksgesundheit und medizinische Forschung (Swiss Life). 


\section{References}

[1] G. Asher, U. Schibler, Crosstalk between components of circadian and metabolic cycles in mammals, Cell Metab, 13 (2011) 125-137.

[2] C. Dibner, U. Schibler, U. Albrecht, The mammalian circadian timing system: organization and coordination of central and peripheral clocks, Annual review of physiology, 72 (2010) 517-549.

[3] K. Eckel-Mahan, P. Sassone-Corsi, Metabolism and the circadian clock converge, Physiol Rev, 93 (2013) 107-135.

[4] B. Feng, T. Zhang, H. Xu, Human adipose dynamics and metabolic health, Ann N Y Acad Sci, 1281 (2013) 160-177.

[5] N. Cybulski, P. Polak, J. Auwerx, M.A. Ruegg, M.N. Hall, mTOR complex 2 in adipose tissue negatively controls whole-body growth, Proc Natl Acad Sci U S A, 106 (2009) 9902-9907.

[6] A. Kumar, J.C. Lawrence, Jr., D.Y. Jung, H.J. Ko, S.R. Keller, J.K. Kim, M.A. Magnuson, T.E. Harris, Fat cell-specific ablation of rictor in mice impairs insulinregulated fat cell and whole-body glucose and lipid metabolism, Diabetes, 59 (2010) 1397-1406.

[7] K. Dragert, I. Bhattacharya, G. Pellegrini, P. Seebeck, A. Azzi, S.A. Brown, S. Georgiopoulou, U. Held, P. Blyszczuk, M. Arras, R. Humar, M.N. Hall, E. Battegay, E. Haas, Deletion of Rictor in brain and fat alters peripheral clock gene expression and increases blood pressure, Hypertension, 66 (2015) 332-339.

[8] M. Laplante, D.M. Sabatini, mTOR signaling in growth control and disease, Cell, 149 (2012) 274-293. 
[9] S. Wullschleger, R. Loewith, M.N. Hall, TOR signaling in growth and metabolism, Cell, 124 (2006) 471-484.

[10] D.D. Sarbassov, S.M. Ali, D.H. Kim, D.A. Guertin, R.R. Latek, H. ErdjumentBromage, P. Tempst, D.M. Sabatini, Rictor, a novel binding partner of mTOR, defines a rapamycin-insensitive and raptor-independent pathway that regulates the cytoskeleton, Curr Biol, 14 (2004) 1296-1302.

[11] M.A. Frias, C.C. Thoreen, J.D. Jaffe, W. Schroder, T. Sculley, S.A. Carr, D.M. Sabatini, mSin1 is necessary for Akt/PKB phosphorylation, and its isoforms define three distinct mTORC2s, Curr Biol, 16 (2006) 1865-1870.

[12] W.J. Oh, E. Jacinto, mTOR complex 2 signaling and functions, Cell Cycle, 10 (2011) 2305-2316.

[13] T.D. Schmittgen, K.J. Livak, Analyzing real-time PCR data by the comparative C(T) method, Nat Protoc, 3 (2008) 1101-1108.

[14] J. Ye, G. Coulouris, I. Zaretskaya, I. Cutcutache, S. Rozen, T.L. Madden, PrimerBLAST: a tool to design target-specific primers for polymerase chain reaction, BMC bioinformatics, 13 (2012) 134.

[15] D.D. Sarbassov, S.M. Ali, S. Sengupta, J.H. Sheen, P.P. Hsu, A.F. Bagley, A.L. Markhard, D.M. Sabatini, Prolonged rapamycin treatment inhibits mTORC2 assembly and Akt/PKB, Mol Cell, 22 (2006) 159-168.

[16] D.A. Guertin, D.M. Stevens, C.C. Thoreen, A.A. Burds, N.Y. Kalaany, J. Moffat, M. Brown, K.J. Fitzgerald, D.M. Sabatini, Ablation in mice of the mTORC components raptor, rictor, or mLST8 reveals that mTORC2 is required for signaling to Akt-FOXO and PKCalpha, but not S6K1, Dev Cell, 11 (2006) 859871. 
[17] M. Lohn, G. Dubrovska, B. Lauterbach, F.C. Luft, M. Gollasch, A.M. Sharma, Periadventitial fat releases a vascular relaxing factor, FASEB J, 16 (2002) 10571063.

[18] E.E. Soltis, L.A. Cassis, Influence of perivascular adipose tissue on rat aortic smooth muscle responsiveness, Clin Exp Hypertens A, 13 (1991) 277-296.

[19] I. Bhattacharya, K. Dragert, V. Albert, E. Contassot, M. Damjanovic, A. Hagiwara, L. Zimmerli, R. Humar, M.N. Hall, E.J. Battegay, E. Haas, Rictor in perivascular adipose tissue controls vascular function by regulating inflammatory molecule expression, Arterioscler Thromb Vasc Biol, 33 (2013) 2105-2111.

[20] M.L. Power, J. Schulkin, Anticipatory physiological regulation in feeding biology: cephalic phase responses, Appetite, 50 (2008) 194-206.

[21] R.D. Rudic, D.J. Fulton, Pressed for time: the circadian clock and hypertension, Journal of applied physiology, 107 (2009) 1328-1338.

[22] W.B. White, Cardiovascular risk and therapeutic intervention for the early morning surge in blood pressure and heart rate, Blood pressure monitoring, 6 (2001) 63-72.

[23] C.B. Anea, M. Zhang, D.W. Stepp, G.B. Simkins, G. Reed, D.J. Fulton, R.D. Rudic, Vascular disease in mice with a dysfunctional circadian clock, Circulation, 119 (2009) 1510-1517.

[24] J.M. Olsen, M. Sato, O.S. Dallner, A.L. Sandstrom, D.F. Pisani, J.C. Chambard, E.Z. Amri, D.S. Hutchinson, T. Bengtsson, Glucose uptake in brown fat cells is dependent on mTOR complex 2-promoted GLUT1 translocation, J Cell Biol, 207 (2014) 365-374.

[25] A. Loboda, W.K. Kraft, B. Fine, J. Joseph, M. Nebozhyn, C. Zhang, Y. He, X. Yang, C. Wright, M. Morris, I. Chalikonda, M. Ferguson, V. Emilsson, A. Leonardson, J. Lamb, H. Dai, E. Schadt, H.E. Greenberg, P.Y. Lum, Diurnal 
variation of the human adipose transcriptome and the link to metabolic disease, BMC Med Genomics, 2 (2009) 7.

[26] A.M. Curtis, M.M. Bellet, P. Sassone-Corsi, L.A. O'Neill, Circadian clock proteins and immunity, Immunity, 40 (2014) 178-186.

[27] T.P. Fitzgibbons, S. Kogan, M. Aouadi, G.M. Hendricks, J. Straubhaar, M.P. Czech, Similarity of mouse perivascular and brown adipose tissues and their resistance to diet-induced inflammation, Am J Physiol Heart Circ Physiol, 301 (2011) H1425-1437.

[28] B.N. Van Vliet, J. McGuire, L. Chafe, A. Leonard, A. Joshi, J.P. Montani, Phenotyping the level of blood pressure by telemetry in mice, Clinical and experimental pharmacology \& physiology, 33 (2006) 1007-1015.

[29] S. Nakashima, Protein kinase C alpha (PKC alpha): regulation and biological function, J Biochem, 132 (2002) 669-675.

[30] Q.W. Fan, W.A. Weiss, Inhibition of PI3K-Akt-mTOR signaling in glioblastoma by mTORC1/2 inhibitors, Methods Mol Biol, 821 (2012) 349-359.

[31] M.M. Morrison, C.D. Young, S. Wang, T. Sobolik, V.M. Sanchez, D.J. Hicks, R.S. Cook, D.M. Brantley-Sieders, mTOR Directs Breast Morphogenesis through the PKC-alpha-Rac1 Signaling Axis, PLoS Genet, 11 (2015) e1005291. 


\section{Figure legends}

Figure 1: Rictor mRNA exhibits a strong diurnal expressional variation in brown adipose adipose tissue and PVAT. (A) EFAT, (B) BAT, (C) PVAT, and (C) aorta were dissected from control and Rictor ${ }^{A P 2 K O}$ mice. Relative mRNA expression levels were measured using quantitative real-time PCR. Values are means \pm SEM, $\mathrm{n}=3$ per group and time point.

Figure 2: Gene expression of $m T O R$ and $m S I N 1$ displays a strong diurnal expressional variation specifically in PVAT. Relative mRNA expression levels of mTOR (A) and mSIN1 (B) were quantified in PVAT and aorta dissected from control mice using quantitative real-time PCR. Values are means \pm SEM, $n=3$ per time point, ${ }^{*} p<0.5$, 2-tailed T-test, n.s.: non significant.

Figure 3: Diurnal variation of basal mTORC2 activity and inflammatory molecule expression in PVAT from control mice. (A) Immunoblots of PVAT extracts obtained from three control mice per time point. ZT refers to Zeitgeber time when control mice were scarified and $M$ to the lane in which protein standard was loaded (left panel). Quantification of total RICTOR and mSIN1 levels and ratio of phosphorylated to total mTORC2 targets AKT and PKCa (right panel). (B) Relative mRNA expression levels of Nos2 and Tnfa were quantified in PVAT using quantitative real-time PCR. Values are means \pm SEM, $n=3,{ }^{*} p<0.05, \quad{ }^{*} * p<0.01$, two tailed T-test. 

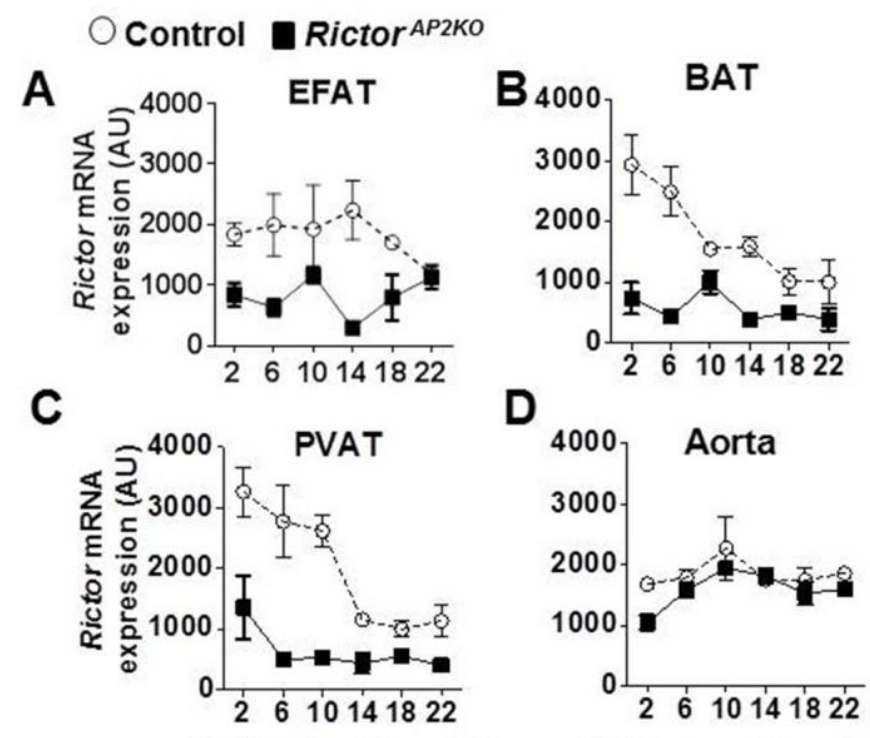

Zeitgeber time (h) Zeitgeber time (h) 
A

PVAT

Aorta
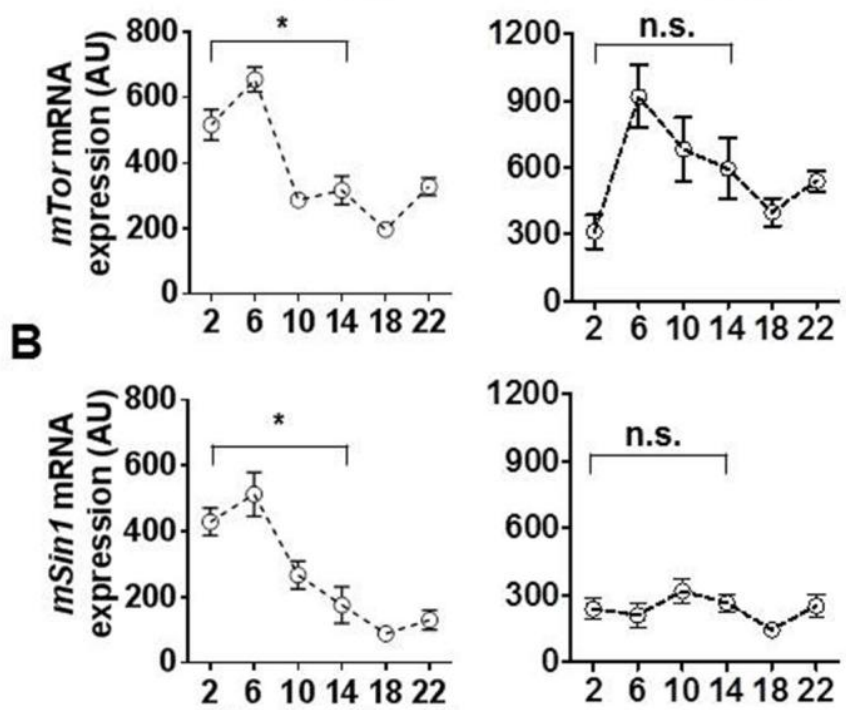

Zeitgeber time (h)

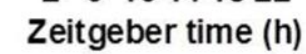




\section{A}

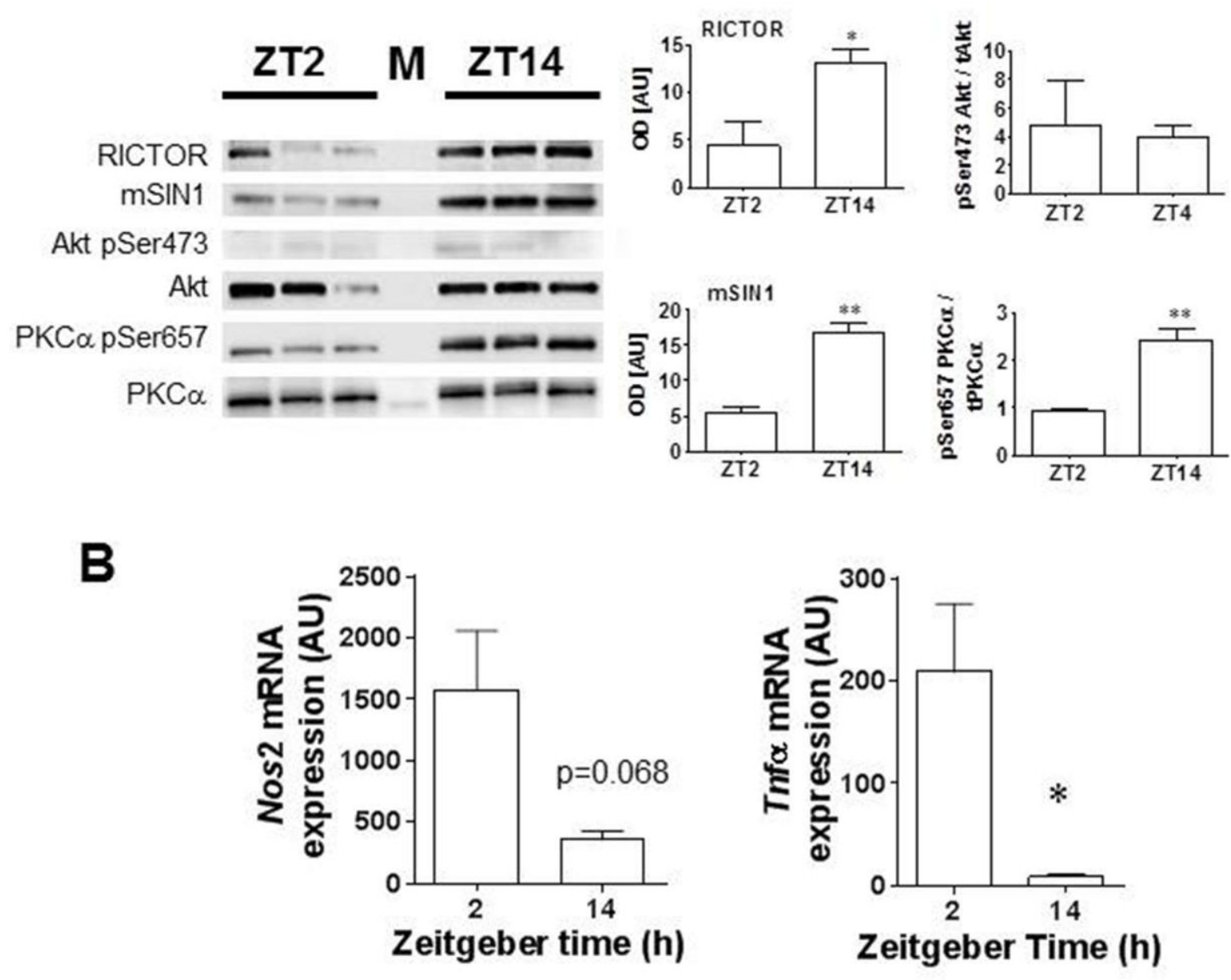


Table 1: Primer pairs used for PCR

\begin{tabular}{lll}
\hline $\begin{array}{l}\text { Gene } \\
\text { symbol }\end{array}$ & Forward Primer (5'-3') & Reverse Primer (5'-3') \\
\hline $\begin{array}{l}\text { min1 } \\
\text { mTor }\end{array}$ & GGGACCCTCCGACCTCTCA & CTCACCTACCTAAGTCACACACC \\
Nos2 & GAAGCCGCGCGAACCT & GGACGCTCACGTTGCTAG AT \\
Rictor & TGCGATATTGGCCATAGTGA & AGCACAGCCACATTGATC \\
Tnfa & GCGGTGCCTATGTCTCAG & GCCATTTGGGAACTTCTCATC \\
Arbp & AGCTGAAGCAAAGGAAGAGTCGGA & ACTTGGTTGCTTTGGCGGGATTAG \\
Cre & GCGGTCTGGCAGTAAAACTATC & ACCCGGCTGCTCTTACTTCT \\
\hline
\end{tabular}


Table 2: Antibodies used for Western Blotting

\begin{tabular}{lll}
\hline Antibody & Company & Dilution \\
\hline rabbit anti AKT & $\begin{array}{l}\text { Cell Signaling \#9272, } \\
\text { Danvers, MA, USA }\end{array}$ & $1: 1000$ \\
$\begin{array}{l}\text { rabbit anti phospho-AKT } \\
\text { (Ser473) }\end{array}$ & $\begin{array}{l}\text { Cell Signaling \#9271, } \\
\text { Danvers, MA, USA }\end{array}$ & $1: 1000$ \\
$\begin{array}{l}\text { rabbit anti phospho- } \\
\text { PKCa (Ser657) }\end{array}$ & $\begin{array}{l}\text { Santa Cruz Biotechnology } \\
\text { \#sC-12356, Dalles, TX, }\end{array}$ & $1: 1000$ \\
USA & \\
rabbit anti PKCa & $\begin{array}{l}\text { Cell Signaling \#2056S, } \\
\text { Danvers, MA, USA }\end{array}$ & $1: 1000$ \\
rabbit anti RICTOR & $\begin{array}{l}\text { Cell Signaling \#2140, } \\
\text { Danvers, MA, USA }\end{array}$ & $1: 1000$ \\
rabbit anti mSIN1 & $\begin{array}{l}\text { abcam \#ab71152, } \\
\text { Cambridge, UK }\end{array}$ & $1: 1000$ \\
anti rabbit HRP & $\begin{array}{l}\text { Amersham Biosciences, } \\
\text { Piscataway, NJ, USA }\end{array}$ & $1: 10000$ \\
\hline
\end{tabular}

